\title{
Ophthalmology Review: A Case-Study Approach by Kuldev Singh et al. (2018) 330pp., 223 illustrations, paperback/softback, ISBN: 9781626231764, Thieme Publishers, New York/Stuttgart
}

\author{
Bradley T. Smith ${ }^{1,2}$
}

Received: 23 February 2019 / Accepted: 3 March 2019 / Published online: 13 March 2019

(C) Springer-Verlag GmbH Germany, part of Springer Nature 2019

Ophthalmology Review: A Case-Study Approach (2nd Edition) is true to its title and geared toward ophthalmology residents. Ninety-eight cases are divided into 11 parts covering Cornea and External Disease, Lens, Glaucoma, Retina, Uveitis, Tumors, Posterior Segment Complications, Trauma, Neuro-Ophthalmology, Pediatrics, and Orbit/Oculoplastics. Complimentary online access is made available at Thieme MedOne. The book includes a multilevel index.

Each topic begins with an abstract of the clinical scenario and themes to be discussed. A detailed case history is presented with quality images. Medical students and new residents should find the descriptions helpful for their own presentations in grand rounds and in the clinic. Discussion of medical and surgical management as well as rehabilitation and followup is included in each case. Though not exhaustive, the motivated learner/reviewer can glean much information by actively interrogating the presentation for pertinent positives and negatives. The "Differential Diagnosis - Key Points" section of each case provides high-yield commentary on each case presentation.

Seasoned attending physicians will likely find the cases stimulate recall of previously learned diagnostic criterion. Clinicians reviewing cases particular to their specialty area (pediatrics, cornea, retina, etc.) will likely note the discussions of therapeutic options are lacking. However, it is appropriate for this work's limited scope as a review text (as disclosed by the authors' preface) and suitable to identify learning gaps motivating the reader toward further study. Suggested supplemental reading is listed after each case presentation.

The book has over 223 images. Few mistakes are present such as redundancy in the pterygium chapter. The chapters on cystoid macular edema and intraocular foreign body have mislabelled images and the right eye zone diagram in the chapter on retinopathy of prematurity shows an inaccurate placement of the fovea. However, the images are of good quality and a strength of this review book.

The case presentation format provides a practical clinical approach delivering on its intent. The lack of dense, bulleted facts makes for a refreshing and digestible review of the most important and frequent encounters in ophthalmology practice. I recommend Ophthalmology Review: A Case-Study Approach to medical students, beginning ophthalmology residents, and physicians who are preparing for their board exams.

Publisher's note Springer Nature remains neutral with regard to jurisdictional claims in published maps and institutional affiliations.
Bradley T. Smith

smithbradleythomas@msn.com

1 The Retina Institute, St. Louis, MO, USA

2 Washington University School of Medicine, Department of Clinical Ophthalmology \& Visual Sciences, St. Louis, MO, USA 